MATHEMATICS OF COMPUTATION

Volume 66, Number 220, October 1997, Pages 1375-1388

S 0025-5718(97)00903-4

\title{
EXTENSION THEOREMS FOR PLATE ELEMENTS WITH APPLICATIONS
}

\author{
JINSHENG GU AND XIANCHENG HU
}

\begin{abstract}
Extension theorems for plate elements are established. Their applications to the analysis of nonoverlapping domain decomposition methods for solving the plate bending problems are presented. Numerical results support our theory.
\end{abstract}

\section{INTRODUCTION}

Consider the plate bending problem with the clamped boundary conditions

$$
\begin{cases}\triangle^{2} u=f & \text { in } \Omega, \\ u=\frac{\partial u}{\partial \nu}=0 & \text { on } \partial \Omega,\end{cases}
$$

where $\Omega \subset \Re^{2}$ is a polygonal domain and $\nu$ the unit outward normal vector. The variational form of (1.1) is

$$
u \in H_{0}^{2}(\Omega): a(u, v)=(f, v), \forall v \in H_{0}^{2}(\Omega),
$$

where

$$
a(u, v)=\int_{\Omega}\left[\triangle u \Delta v+(1-\gamma)\left(2 \partial_{12} u \partial_{12} v-\partial_{11} u \partial_{22} v-\partial_{22} u \partial_{11} v\right)\right] d x,
$$

$(f, v)=\int_{\Omega} f v d x$ and $\gamma \in(0,0.5)$ is the Poisson ratio. As is well-known, the unique solvability of (1.2) for $f \in L^{2}(\Omega)$ follows from the continuity and coerciveness of the bilinear form $a(\cdot, \cdot)$ in $H_{0}^{2}(\Omega)$ (cf. [7], [9], [19] for details).

Suppose that $\Omega_{h}=\{e\}$ is a quasi-uniform mesh of $\Omega$, i.e., $\Omega_{h}$ satisfies

$$
\sup _{e \in \Omega_{h}} \inf _{B_{r} \supset e} r \leq c h, \quad \inf _{e \in \Omega_{h}} \sup _{B_{r} \subset e} r \geq C h,
$$

where $e$, a triangle, represents the typical element in $\Omega_{h}, B_{r}$ is a region bounded by the circle of radius $r, h=\max _{e \in \Omega_{h}} h_{e}$ is the mesh parameter and $h_{e}=\inf _{B_{r} \supset e} r$. Here and later, $c$ and $C$ denote generic positive constants independent of $h$. Let $V_{h}$ be the Morley nonconforming finite element space $[18,21]$ associated with $\Omega_{h}$. Then $v \in V_{h}$ if and only if it has the following three properties:

(1) $\left.v\right|_{e}$ is quadratic, $\forall e \in \Omega_{h}$;

(2) $v$ is continuous at each vertex $p$ of $e, \forall e \in \Omega_{h}$;

Received by the editor November 22, 1994 and, in revised form, November 22, 1995 and May $1,1996$.

1991 Mathematics Subject Classification. Primary 65F10, 65N30.

Key words and phrases. Extension theorem, plate element, domain decomposition.

This work was supported by the National Natural Science Foundation of China. 
(3) $\frac{\partial v}{\partial \nu}$ is continuous at each edge midpoint $m$ of $e, \forall e \in \Omega_{h}$.

Throughout this paper, we let $p$ and $m$ (with or without subscripts) denote a vertex and an edge midpoint of the elements in $\Omega_{h}$ respectively. The Morley element discrete problem of $(1.2)$ is

$$
u_{h} \in V_{h}^{0}: A\left(u_{h}, v\right)=(f, v), \forall v \in V_{h}^{0},
$$

where

$$
\begin{gathered}
V_{h}^{0}=\left\{v \in V_{h}: v(p)=0, \frac{\partial v}{\partial \nu}(m)=0, \forall p, m \in \partial \Omega\right\} \\
A(w, v)=\sum_{e \in \Omega_{h}} \int_{e}\left[\triangle w \triangle v+(1-\gamma)\left(2 \partial_{12} w \partial_{12} v-\partial_{11} w \partial_{22} v-\partial_{22} w \partial_{11} v\right)\right] d x
\end{gathered}
$$

Some progress has been made in the research of domain decomposition methods for (1.2). Chan et al. [5] presented interface preconditioners for the biharmonic equations via the finite difference methods where the interface consisits of two grid lines, while Sun [23] constructed the multilevel preconditioners for the biharmonic equations via the $\mathrm{B}$-spline methods. All their methods require that the domain $\Omega$ should be a rectangle. Brenner [4] proposed a two-level additive Schwarz preconditioner for nonconforming plate elements through intergrid transfer operators. Gu [10] considered the parallel Schwarz alternating algorithm for (1.4) and found the preconditioner of the algorithm by employing the idea of Widlund [8]. Zhang [25][27] and Oswald [20] have recently studied hierarchical, multilevel and Schwarz methods for discretizations of the biharmonic equation by conforming finite elements.

The purpose of this paper is to give the extension theorem for Morley elements with applications to solving (1.4) and further to point out that the extension theorems for other plate elements $[4,6]$ hold. It is known that the extension theorems play key roles in the analysis of nonoverlapping domain decomposition methods for the second order elliptic problems discretized by the conforming or nonconforming finite element methods $[10,11,14,24]$. When considering the nonoverlapping domain decomposition methods for the solving of (1.4), we must establish the extension theorem correspondingly. To this end, the conforming interpolation operator introduced in [4] is modified to act as a bridge between Morley nonconforming element space and Argyris conforming element space [2], and its stability proof is presented thereafter. Additionally we estimate the error of the Morley element approximate solution of the inhomogeneous boundary value problem under the weak condition that the solution of (1.2), $u \in H^{3}(\Omega)$. Hence the extension theorem for Morley elements is established eventually. To illustrate its applications, we describe and analyze a nonoverlapping domain decomposition algorithm with two subdomains. In each iteration of this algorithm, the solution of a discrete subproblem on one subdomain with the Dirichlet condition on the interface is followed by the solution of a discrete subproblem on another subdomain with the Neumann condition on the interface. So it is in fact the generalization of the Dirichlet-Neumann alternating method (also known as the Marini-Quarteroni algorithm [17]). Based on the extension theorem, we show that it is geometrically convergent and the convergence factor independent of $h$. Numerical results are also presented to indicate that the theoretical estimate is fully realized in practice. It is more important that via the same idea as above, we eventually obtain the extension theorems for all the conforming plate elements [6] and for other nonconforming plate elements [4]. 
The remainder of this paper is organized as follows. In $\S 2$, we describe and prove Theorem 2.4, the extension theorem for Morley elements. Its applications to the analysis of nonoverlapping domain decomposition methods and numerical experiments are given in $\S 3$. To conclude the paper, we point out in $\S 4$ that the extension theorems for other plate elements hold.

\section{Extension theOREM FOR MORLEy ELEMENTS}

The trace estimates are important tools in many nonconforming finite element analyses. For our purpose, a simple one is stated as follows

Lemma $2.1([13,16])$. If $e$ is affine equivalent to the reference element $\hat{e}$, then

$$
\int_{\partial e} w^{2} d s \leq c\left\{h_{e}^{-1}\|w\|_{0, e}^{2}+h_{e}|w|_{1, e}^{2}\right\}, \forall w \in H^{1}(e) .
$$

Theorem 2.2. Let $\tilde{\Gamma} \subset \partial \Omega$ be an open edge of a polygonal domain $\Omega$. Suppose the functions $g_{1}, g_{2}$ defined on $\partial \Omega$ satisfy $\left.g_{1}\right|_{\tilde{\Gamma}} \in H_{00}^{\frac{5}{2}}(\tilde{\Gamma}),\left.g_{2}\right|_{\tilde{\Gamma}} \in H_{00}^{\frac{3}{2}}(\tilde{\Gamma}),\left.g_{1}\right|_{\partial \Omega \backslash \tilde{\Gamma}}=$ $\left.g_{2}\right|_{\partial \Omega \backslash \tilde{\Gamma}}=0$. Let $\theta \in H^{3}(\Omega), \theta_{h} \in V_{h}$, be respectively the solutions of the following problems:

$$
\left\{\begin{array} { l l } 
{ a ( \theta , v ) = 0 , } & { \forall v \in H _ { 0 } ^ { 2 } ( \Omega ) , } \\
{ \theta = g _ { 1 } , } & { \text { on } \partial \Omega , } \\
{ \frac { \partial \theta } { \partial \nu } = g _ { 2 } , } & { \text { on } \partial \Omega , }
\end{array} \quad \left\{\begin{array}{ll}
A\left(\theta_{h}, v\right)=0, & \forall v \in V_{h}^{0}, \\
\theta_{h}(p)=g_{1}(p), & \forall p \in \partial \Omega, \\
\frac{\partial \theta_{h}}{\partial \nu}(m)=g_{2}(m), & \forall m \in \partial \Omega .
\end{array}\right.\right.
$$

Then we have

$$
\left|\theta-\theta_{h}\right|_{h, \Omega} \leq \operatorname{ch}|\theta|_{H^{3}(\Omega)},
$$

where $|w|_{h, \Omega} \triangleq\left(\sum_{e \subset \Omega}|w|_{2, e}^{2}\right)^{\frac{1}{2}}$.

Proof. Denote $V_{h}^{*}=\left\{v \in V_{h}: v(p)=\theta(p), \frac{\partial v}{\partial \nu}(m)=\frac{\partial \theta}{\partial \nu}(m), \forall p, m \in \partial \Omega\right\}$. $\forall v \in V_{h}^{*}$, it is easy to see that

$$
\begin{aligned}
c\left|\theta_{h}-v\right|_{h, \Omega}^{2} & \leq A\left(\theta_{h}-v, \theta_{h}-v\right) \\
& =A\left(\theta-v, \theta_{h}-v\right)+A\left(\theta_{h}, \theta_{h}-v\right)-A\left(\theta, \theta_{h}-v\right) \\
& \leq c|\theta-v|_{h, \Omega}\left|\theta_{h}-v\right|_{h, \Omega}+0+\left|A\left(\theta, \theta_{h}-v\right)\right| .
\end{aligned}
$$

Hence

$$
\begin{aligned}
\left|\theta_{h}-v\right|_{h, \Omega} & \leq c\left\{|\theta-v|_{h, \Omega}+\frac{\left|A\left(\theta, \theta_{h}-v\right)\right|}{\left|\theta_{h}-v\right|_{h, \Omega}}\right\} \\
& \leq c\left\{|\theta-v|_{h, \Omega}+\sup _{w \in V_{h}^{0}} \frac{|A(\theta, w)|}{|w|_{h, \Omega}}\right\}, \forall v \in V_{h}^{*} .
\end{aligned}
$$

By the triangle inequality, we get

$$
\left|\theta-\theta_{h}\right|_{h, \Omega} \leq c\left(\inf _{v \in V_{h}^{*}}|\theta-v|_{h, \Omega}+\sup _{w \in V_{h}^{0}} \frac{|A(\theta, w)|}{|w|_{h, \Omega}}\right) .
$$

(2.1) is in fact a variant of the second Strang lemma [6] in the nonhomogeneous boundary value case. 
Let $w \in V_{h}^{0}$. Applying Green's formula yields

$$
A(\theta, w)=-\sum_{e \subset \Omega} \int_{e} \nabla(\triangle \theta) \cdot \nabla w d x+E_{1}(\theta, w)+E_{2}(\theta, w),
$$

where

$$
\begin{gathered}
E_{1}(\theta, w)=(1-\gamma) \sum_{e \subset \Omega} \int_{\partial e} \frac{\partial^{2} \theta}{\partial \nu \partial s} \frac{\partial w}{\partial s} d s \\
E_{2}(\theta, w)=\sum_{e \subset \Omega} \int_{\partial e}\left[\Delta \theta-(1-\gamma) \frac{\partial^{2} \theta}{\partial s^{2}}\right] \frac{\partial w}{\partial \nu} d s .
\end{gathered}
$$

Denote

$$
\mathcal{D}(\Omega)=\left\{v \in \mathcal{C}^{\infty}(\Omega): \operatorname{supp} v \text { is a compact subset of } \Omega\right\}
$$

We note that $\theta$ satisfies

$$
\sum_{e \subset \Omega} \int_{e} \nabla(\triangle \theta) \cdot \nabla v d x=\int_{\Omega} \nabla(\triangle \theta) \cdot \nabla v d x=-\int_{\Omega} \triangle \theta \triangle v d x=0, \forall v \in \mathcal{D}(\Omega) .
$$

Since $\mathcal{D}(\Omega)$ is dense in $H_{0}^{1}(\Omega)$, we have

$$
\begin{gathered}
\sum_{e \subset \Omega} \int_{e} \nabla(\triangle \theta) \cdot \nabla v d x=0, \forall v \in H_{0}^{1}(\Omega), \\
A(\theta, w)=\sum_{e \subset \Omega} \int_{e} \nabla(\triangle \theta) \cdot \nabla\left(L_{e} w-w\right) d x+E_{1}(\theta, w)+E_{2}(\theta, w),
\end{gathered}
$$

where $L_{e}$ is the linear interpolation operator on $e$ with the vertices of $e$ as interpolation points.

Three notations: $M_{e}, M_{F}$ and $\alpha$ are used throughout the remainder of this section in the following sense. $M_{e}$ is the mean value operator over the element $e$, defined by

$$
M_{e} v=\frac{1}{\operatorname{meas}(e)} \int_{e} v d x, \quad \forall v \in L^{2}(e),
$$

while $M_{F}$ is the mean value operator over the edge $F$ of $e$ which can be defined similarly. $\alpha=\left(\alpha_{1}, \alpha_{2}\right) \in \mathbf{N}^{2}$ is a multi-index with $|\alpha| \triangleq \alpha_{1}+\alpha_{2}$. For example, if $\alpha=(1,2)$, then $|\alpha|=3$ and $\partial_{\alpha}=\frac{\partial^{3}}{\partial x \partial y^{2}}$.

For each edge $F$, if $F=\partial e \cap \partial \Omega$ for some $e$, it is obvious that $\frac{\partial}{\partial s}\left(L_{e} w\right)=0$; if $F=\partial e_{1} \cap \partial e_{2}$ for some elements $e_{1}$ and $e_{2}$, then $\left.\frac{\partial}{\partial s}\left(L_{e_{1}} w\right)\right|_{F}=\left.\frac{\partial}{\partial s}\left(L_{e_{2}} w\right)\right|_{F}$. Furthermore, for each $e, \int_{\partial e} \frac{\partial\left(w-L_{e} w\right)}{\partial s} d s=0$. By the Schwarz inequality, 
Lemma 2.1, interpolation error estimates [6] and inverse inequalities [6], we obtain

$$
\begin{aligned}
& E_{1}(\theta, w)=(1-\gamma) \sum_{e \subset \Omega} \int_{\partial e}\left[\frac{\partial^{2} \theta}{\partial \nu \partial s}-M_{e}\left(\frac{\partial^{2} \theta}{\partial \nu \partial s}\right)\right] \frac{\partial\left(w-L_{e} w\right)}{\partial s} d s \\
& \leq(1-\gamma)\left(\sum_{e \subset \Omega} \int_{\partial e}\left|\frac{\partial^{2} \theta}{\partial \nu \partial s}-M_{e}\left(\frac{\partial^{2} \theta}{\partial \nu \partial s}\right)\right|^{2} d s\right)^{\frac{1}{2}}\left(\sum_{e \subset \Omega} \int_{\partial e}\left|\frac{\partial\left(w-L_{e} w\right)}{\partial s}\right|^{2} d s\right)^{\frac{1}{2}} \\
& \leq c\left(\sum_{e \subset \Omega} \sum_{|\alpha|=2} \int_{\partial e}\left|\partial_{\alpha} \theta-M_{e}\left(\partial_{\alpha} \theta\right)\right|^{2} d s\right)^{\frac{1}{2}}\left(\sum_{e \subset \Omega} \sum_{|\alpha|=1} \int_{\partial e}\left|\partial_{\alpha}\left(w-L_{e} w\right)\right|^{2} d s\right)^{\frac{1}{2}} \\
& \leq c\left(\sum_{e \subset \Omega} \sum_{|\alpha|=2}\left[h_{e}^{-1}\left\|\partial_{\alpha} \theta-M_{e}\left(\partial_{\alpha} \theta\right)\right\|_{0, e}^{2}+h_{e}\left|\partial_{\alpha} \theta-M_{e}\left(\partial_{\alpha} \theta\right)\right|_{1, e}^{2}\right]\right)^{\frac{1}{2}} \\
& \cdot\left(\sum_{e \subset \Omega|\alpha|=1}\left[h_{e}^{-1}\left\|\partial_{\alpha}\left(w-L_{e} w\right)\right\|_{0, e}^{2}+h_{e}\left|\partial_{\alpha}\left(w-L_{e} w\right)\right|_{1, e}^{2}\right)^{\frac{1}{2}}\right. \\
& \leq c\left(\sum_{e \subset \Omega} \sum_{|\alpha|=2} h_{e}\left|\partial_{\alpha} \theta\right|_{1, e}^{2}\right)^{\frac{1}{2}}\left(\sum_{e \subset \Omega} \sum_{|\alpha|=1} h_{e}\left|\partial_{\alpha} w\right|_{1, e}^{2}\right)^{\frac{1}{2}} \\
& \leq c h|\theta|_{3, \Omega}|w|_{h, \Omega} .
\end{aligned}
$$

We notice that for each edge $F$, if $F \subset \partial \Omega$, it is obvious that $M_{F}\left(\frac{\partial w}{\partial \nu}\right)=0$; if $F=\partial e_{1} \cap \partial e_{2}$ for some elements $e_{1}$ and $e_{2}$, then $\sum_{i=1}^{2} M_{F}\left(\left.\frac{\partial w}{\partial \nu_{i}}\right|_{F \subset \partial e_{i}}\right)=0$, where $\nu_{i}$ is the unit outward normal vector of $e_{i}$. In addition, $\int_{F}\left[\frac{\partial w}{\partial \nu}-M_{F}\left(\frac{\partial w}{\partial \nu}\right)\right] d s=0$ for each edge $F$ and $\int_{F}\left(\phi-M_{F} \phi\right)^{2} d s \leq \int_{F}(\phi-\beta)^{2} d s$ for any measurable function $\phi$ and any constant $\beta \in \Re$. In the same manner as above, we have

$$
\begin{aligned}
E_{2}(\theta, w)= & \sum_{e \subset \Omega} \sum_{F \subset \partial e} \int_{F}\left[\triangle \theta-(1-\gamma) \frac{\partial^{2} \theta}{\partial s^{2}}-M_{F}\left(\triangle \theta-(1-\gamma) \frac{\partial^{2} \theta}{\partial s^{2}}\right)\right] \\
& \cdot\left[\frac{\partial w}{\partial \nu}-M_{F}\left(\frac{\partial w}{\partial \nu}\right)\right] d s \\
\leq & c h|\theta|_{3, \Omega}|w|_{h, \Omega} .
\end{aligned}
$$

Let $\pi_{h}$ be the interpolation operator of the Morley element space $V_{h}$. Then $\pi_{h} \theta \in V_{h}^{*}$. The standard interpolation error estimate gives

$$
\begin{gathered}
\sum_{e \subset \Omega} \int_{e} \nabla(\triangle \theta) \cdot \triangle\left(w-L_{e} w\right) d x \leq \operatorname{ch|}|\theta|_{3, \Omega}|w|_{h, \Omega}, \\
\inf _{v \in V_{h}^{*}}|\theta-v|_{h, \Omega} \leq\left|\theta-\pi_{h} \theta\right|_{h, \Omega} \leq \operatorname{ch}|\theta|_{3, \Omega} .
\end{gathered}
$$

By using (2.1), (2.2) and the arguments that followed, we can complete the proof of the theorem.

Suppose that there exists an open straight line, $\Gamma$, which divides $\Omega$ into two open convex subdomains $\Omega_{1}$ and $\Omega_{2}$ s.t. $\Omega=\Omega_{1} \cup \Omega_{2} \cup \Gamma, \Omega_{1} \cap \Omega_{2}=\emptyset, \Gamma \cap e=\emptyset, \forall e \in \Omega_{h}$. For $k=1,2$, denote

$$
|w|_{h, \Omega_{k}} \triangleq\left(\sum_{e \subset \Omega_{k}}|w|_{2, e}^{2}\right)^{\frac{1}{2}}, \quad(w, v)_{k}=\int_{\Omega_{k}} w v d x
$$




$$
\begin{gathered}
V_{h}^{k}=\left\{v \in V_{h}^{0}: v(p)=0, \frac{\partial v}{\partial \nu}(m)=0, \forall p, m \in \Omega \backslash \bar{\Omega}_{k}\right\}, \\
V_{h}^{k, 0}=\left\{v \in V_{h}^{0}: v(p)=0, \frac{\partial v}{\partial \nu}(m)=0, \forall p, m \in \Omega \backslash \Omega_{k}\right\}, \\
A_{k}(w, v)=\sum_{e \subset \Omega_{k}} \int_{e}\left[\triangle w \triangle v+(1-\gamma)\left(2 \partial_{12} w \partial_{12} v-\partial_{11} w \partial_{22} v-\partial_{22} w \partial_{11} v\right)\right] d x .
\end{gathered}
$$

Brenner [4] has introduced an interpolation operator $I_{h}^{k}$ which acts as a bridge between the Morley nonconforming element space $V_{h}^{k}$ and the Argyris conforming element [2] space $\mathcal{A} R_{h}^{k}$. Here, $v \in \mathcal{A} R_{h}^{k}$ if and only if $v$ satisfies

(1) $\left.v\right|_{e}$ is a fifth order polynomial, $\forall e \subset \Omega_{k}$;

(2) $\partial_{\alpha} v(0 \leq|\alpha| \leq 2)$ are continuous at each vertex $p$ of $e, \forall e \subset \Omega_{k}$;

(3) $\frac{\partial v}{\partial \nu}$ is continuous at each edge midpoint $m$ of $e, \forall e \subset \Omega_{k}$;

(4) $\partial_{\alpha} v(p)=0,(0 \leq|\alpha| \leq 2), \frac{\partial v}{\partial \nu}(m)=0, \forall p, m \in \partial \Omega_{k} \backslash \Gamma$.

For our purpose, we modify $I_{h}^{k}$ as follows: $\forall v \in V_{h}^{k}, I_{h}^{k} v \in \mathcal{A} R_{h}^{k}$ s.t.

1. $\left(I_{h}^{k} v\right)(p)=v(p)$,

2. $\frac{\partial\left(I_{h}^{k} v\right)}{\partial \nu}(m)=\frac{\partial v}{\partial \nu}(m)$,

3. $\left[\partial_{\alpha}\left(I_{h}^{k} v\right)\right](p)=0, \quad|\alpha|=2$,

4. $\quad\left[\partial_{\alpha}\left(I_{h}^{k} v\right)\right](p)= \begin{cases}0, & |\alpha|=1, p \in \partial \Omega_{k} \backslash \Gamma, \\ \text { average of }\left(\partial_{\alpha} v_{i}\right)(p), & |\alpha|=1, p \notin \partial \Omega_{k} \backslash \Gamma,\end{cases}$

where $\left.v_{i} \triangleq v\right|_{e_{i}}$ and $e_{i}$ contains $p$ as a vertex.

Theorem 2.3. If $I_{h}^{k}$ is defined as above, then

$$
\begin{gathered}
I_{h}^{k} v=\frac{\partial\left(I_{h}^{k} v\right)}{\partial \nu}=0 \quad \text { on } \partial \Omega_{k} \backslash \Gamma, \quad \forall v \in V_{h}^{k}, \\
\left\|v-I_{h}^{k} v\right\|_{L^{2}\left(\Omega_{k}\right)} \leq c h^{2}|v|_{h, \Omega_{k}}, \forall v \in V_{h}^{k} .
\end{gathered}
$$

Proof. (2.5) follows from the definition of $I_{h}^{k}$. (2.6) can be obtained by modifying the proof of Lemma 5.1 [4]. For completeness, we outline it as follows.

Let $v \in V_{h}^{k}$ and $e \subset \Omega_{k}$. Denote $w=\left.v\right|_{e}$ and $\tilde{w}=\left.\left(I_{h}^{k} v\right)\right|_{e}$. Then

$$
w-\tilde{w}=\sum_{i=1}^{3} \sum_{|\alpha|=1,2} \partial_{\alpha}(w-\tilde{w})\left(p_{i}\right) r_{\alpha, i},
$$

where the functions $r_{\alpha, i}$ are the nodal basis functions corresponding to the nodal variables $\left(\partial_{\alpha} v\right)\left(p_{i}\right)$ of the Argyris element space on $e$.

By standard techniques of the almost affine-equivalent family of finite elements [6], we see that

$$
\begin{aligned}
& \left\|r_{\alpha, i}\right\|_{L^{2}(e)} \leq c h^{2} \quad \text { for }|\alpha|=1 \\
& \left\|r_{\alpha, i}\right\|_{L^{2}(e)} \leq c h^{3} \quad \text { for }|\alpha|=2
\end{aligned}
$$

If $|\alpha|=2$, then

$$
\left|\partial_{\alpha}(w-\tilde{w})\left(p_{i}\right)\right|=\left|\partial_{\alpha} w\left(p_{i}\right)\right| \leq|v|_{W_{\infty}^{2}(e)} \leq c h^{-1}|v|_{2, e} .
$$

We next discuss the case that $|\alpha|=1$.

Suppose that $p_{1} \in \partial \Omega_{k} \backslash \Gamma$. Since $\Omega_{h}$ is quasi-uniform, there exists a positive integer $J$, independent of $h$, such that $e_{1}, e_{2}, \cdots, e_{J}=e \subset \Omega_{k}, e_{1}, e_{2}, \cdots, e_{J}$ 
contain $p_{1}$ as a common vertex, meas $\left(\partial e_{j} \cap \partial e_{j+1}\right)>0$ for $j=1,2, \cdots, J-1$ and $\operatorname{meas}\left(\partial e_{1} \cap\left(\partial \Omega_{k} \backslash \Gamma\right)\right)>0$.

By Taylor's formula and the fact that $\left.v\right|_{e_{j}}$ and $\left.v\right|_{e_{j-1}}$ agree at the two endpoints of $\partial e_{j} \cap \partial e_{j-1}$, it is easy to obtain

$$
\left|\frac{\partial\left(\left.v\right|_{e_{j}}\right)}{\partial s}\left(p_{1}\right)-\frac{\partial\left(\left.v\right|_{e_{j-1}}\right)}{\partial s}\left(p_{1}\right)\right| \leq \frac{h}{2}\left[|v|_{W_{\infty}^{2}\left(e_{j}\right)}+|v|_{W_{\infty}^{2}\left(e_{j-1}\right)}\right]
$$

where $s$ is the arc length along $\partial e_{j} \cap \partial e_{j-1}$. Similarly since $\frac{\partial\left(\left.v\right|_{e_{j}}\right)}{\partial \nu}$ and $\frac{\partial\left(\left.v\right|_{e_{j-1}}\right)}{\partial \nu}$ agree at the midpoint of $\partial e_{j} \cap \partial e_{j-1}$, we get

$$
\left|\frac{\partial\left(\left.v\right|_{e_{j}}\right)}{\partial \nu}\left(p_{1}\right)-\frac{\partial\left(\left.v\right|_{e_{j-1}}\right)}{\partial \nu}\left(p_{1}\right)\right| \leq \frac{h}{2}\left[|v|_{W_{\infty}^{2}\left(e_{j}\right)}+|v|_{W_{\infty}^{2}\left(e_{j-1}\right)}\right] .
$$

Therefore, we have

$$
\left|\partial_{\alpha}\left(\left.v\right|_{e_{j}}\right)\left(p_{1}\right)-\partial_{\alpha}\left(\left.v\right|_{e_{j-1}}\right)\left(p_{1}\right)\right| \leq c h\left[|v|_{W_{\infty}^{2}\left(e_{j}\right)}+|v|_{W_{\infty}^{2}\left(e_{j-1}\right)}\right] .
$$

Let $p_{1}^{\prime} \in \partial e_{1} \cap\left(\partial \Omega_{k} \backslash \Gamma\right)$ be another endpoint of the edge $\partial e_{1} \cap\left(\partial \Omega_{k} \backslash \Gamma\right)$. Since $v\left(p_{1}\right)=v\left(p_{1}^{\prime}\right)=0$, there exists a point $q \in \partial e_{1} \cap\left(\partial \Omega_{k} \backslash \Gamma\right)$, s.t. $\frac{\partial\left(\left.v\right|_{e_{1}}\right)}{\partial s}(q)=0$. Obviously, $\frac{\partial\left(v e_{1}\right)}{\partial \nu}\left(m_{1}\right)=0$, where $m_{1}$ is the midpoint of the edge $\partial e_{1} \cap\left(\partial \Omega_{k} \backslash \Gamma\right)$. Then

$$
\begin{gathered}
\left|\frac{\partial\left(\left.v\right|_{e_{1}}\right)}{\partial s}\left(p_{1}\right)\right|=\left|\frac{\partial\left(\left.v\right|_{e_{1}}\right)}{\partial s}\left(p_{1}\right)-\frac{\partial\left(\left.v\right|_{e_{1}}\right)}{\partial s}(q)\right| \leq h|v|_{W_{\infty}^{2}\left(e_{1}\right)}, \\
\left|\frac{\partial\left(\left.v\right|_{e_{1}}\right)}{\partial \nu}\left(p_{1}\right)\right|=\left|\frac{\partial\left(\left.v\right|_{e_{1}}\right)}{\partial \nu}\left(p_{1}\right)-\frac{\partial\left(\left.v\right|_{e_{1}}\right)}{\partial \nu}\left(m_{1}\right)\right| \leq h|v|_{W_{\infty}^{2}\left(e_{1}\right)} .
\end{gathered}
$$

So $\left|\partial_{\alpha}\left(\left.v\right|_{e_{1}}\right)\left(p_{1}\right)\right| \leq c h|v|_{W_{\infty}^{2}\left(e_{1}\right)}$.

$$
\begin{aligned}
\left|\partial_{\alpha}(w-\tilde{w})\left(p_{1}\right)\right| & =\left|\partial_{\alpha}\left(\left.v\right|_{e}\right)\left(p_{1}\right)\right| \\
& =\left|\sum_{j=2}^{J}\left[\partial_{\alpha}\left(\left.v\right|_{e_{j}}\right)\left(p_{1}\right)-\partial_{\alpha}\left(\left.v\right|_{e_{j-1}}\right)\left(p_{1}\right)\right]+\partial_{\alpha}\left(\left.v\right|_{e_{1}}\right)\left(p_{1}\right)\right| \\
& \leq \sum_{j=2}^{J}\left|\partial_{\alpha}\left(\left.v\right|_{e_{j}}\right)\left(p_{1}\right)-\partial_{\alpha}\left(\left.v\right|_{e_{j-1}}\right)\left(p_{1}\right)\right|+\left|\partial_{\alpha}\left(\left.v\right|_{e_{1}}\right)\left(p_{1}\right)\right| \\
& \leq c h \sum_{j=1}^{J}|v|_{W_{\infty}^{2}\left(e_{j}\right)} \leq c \sum_{j=1}^{J}|v|_{2, e_{j}} \leq c \sum_{e^{\prime}}|v|_{2, e^{\prime}},
\end{aligned}
$$

where $e^{\prime} \subset \Omega_{k}$ s.t. $\partial e^{\prime} \cap \partial e \neq \emptyset$.

If $p_{1} \notin \partial \Omega_{k} \backslash \Gamma$, then by the same argument as above, we can easily obtain

$$
\left|\partial_{\alpha}(w-\tilde{w})\left(p_{1}\right)\right| \leq c \sum_{e^{\prime}}|v|_{2, e^{\prime}}
$$

Therefore, we have

$$
\left\|v-I_{h}^{k} v\right\|_{L^{2}(e)} \leq c h^{2} \sum_{e^{\prime}}|v|_{2, e^{\prime}} .
$$

Summing up the square of the last inequality over all the elements $e \subset \Omega_{k}$, we eventually get (2.6) by the quasi-uniformness of the mesh $\Omega_{h}$.

In what follows, $\left\{p_{i}\right\}_{i=1}^{I}$ denotes the set of the vertices on $\Gamma$ and $\left\{m_{j}\right\}_{j=1}^{J}$ the set of the edge midpoints on $\Gamma$. Let $\nu_{k}(k=1,2)$ be the unit outward normal vector 
of $\Omega_{k} . r_{0}: V_{h} \rightarrow \Re^{I}$ and $r_{1}: V_{h} \rightarrow \Re^{J}$ denote respectively the discrete operators such that

$$
\begin{gathered}
\forall v \in V_{h}, r_{0} v \in \Re^{I}:\left(r_{0} v\right)(i)=v\left(p_{i}\right), i=1,2, \cdots, I ; \\
\forall w \in V_{h}, r_{1} w \in \Re^{J}:\left(r_{1} w\right)(j)=\frac{\partial w}{\partial \nu_{1}}\left(m_{j}\right), j=1,2, \cdots, J .
\end{gathered}
$$

Define the discrete biharmonic extension operator $E_{h}^{k}: \Re^{I} \times \Re^{J} \rightarrow V_{h}^{k}$ as follows:

$$
\forall(\lambda, \mu) \in \Re^{I} \times \Re^{J}, E_{h}^{k}(\lambda, \mu) \in V_{h}^{k}:\left\{\begin{array}{l}
A_{k}\left(E_{h}^{k}(\lambda, \mu), v\right)=0, \quad \forall v \in V_{h}^{k, 0}, \\
r_{0} E_{h}^{k}(\lambda, \mu)=\lambda, \\
r_{1} E_{h}^{k}(\lambda, \mu)=\mu .
\end{array}\right.
$$

Theorem 2.4. (Extension theorem for Morley elements) If $\Omega_{1}, \Omega_{2}$ are convex polygonal domains, there exist two constants $\sigma, \tau$, independent of the quasi-uniform mesh parameter $h$, such that

$$
\begin{gathered}
\sigma=\sup _{(\lambda, \mu) \in \Re^{I} \times \Re^{J}} \frac{A_{1}\left(E_{h}^{1}(\lambda, \mu), E_{h}^{1}(\lambda, \mu)\right)}{A_{2}\left(E_{h}^{2}(\lambda, \mu), E_{h}^{2}(\lambda, \mu)\right)}<\infty \\
\tau=\sup _{(\lambda, \mu) \in \Re^{I} \times \Re^{J}} \frac{A_{2}\left(E_{h}^{2}(\lambda, \mu), E_{h}^{2}(\lambda, \mu)\right)}{A_{1}\left(E_{h}^{1}(\lambda, \mu), E_{h}^{1}(\lambda, \mu)\right)}<\infty
\end{gathered}
$$

Proof. Let $(\lambda, \mu) \in \Re^{I} \times \Re^{J}$. Denote $u_{k}^{h}=E_{h}^{k}(\lambda, \mu)$ for convenience. With the inverse inequality and Theorem 2.3, we have

$$
\left|u_{1}^{h}-I_{h}^{1} u_{1}^{h}\right|_{h, \Omega_{1}}^{2}=\sum_{e \subset \Omega_{1}}\left|u_{1}^{h}-I_{h}^{1} u_{1}^{h}\right|_{2, e}^{2} \leq c h^{-4}\left\|u_{1}^{h}-I_{h}^{1} u_{1}^{h}\right\|_{L^{2}\left(\Omega_{1}\right)}^{2} \leq c\left|u_{1}^{h}\right|_{h, \Omega_{1}}^{2} .
$$

Therefore, $I_{h}^{1} u_{1}^{h} \in \mathcal{A} R_{h}^{1} \subset H^{2}\left(\Omega_{1}\right)$ and the triangle inequality yields

$$
\left|I_{h}^{1} u_{1}^{h}\right|_{H^{2}\left(\Omega_{1}\right)}=\left|I_{h}^{1} u_{1}^{h}\right|_{h, \Omega_{1}} \leq c\left|u_{1}^{h}\right|_{h, \Omega_{1}} .
$$

Furthermore, applying the trace theorem and the Poincaré-Friedrichs inequality in $H^{2}\left(\Omega_{1}\right)$ gives

$$
\begin{aligned}
& \left\|I_{h}^{1} u_{1}^{h}\right\|_{H_{00}^{\frac{3}{2}}(\Gamma)}^{2}+\left\|\frac{\partial\left(I_{h}^{1} u_{1}^{h}\right)}{\partial \nu_{1}}\right\|_{H_{00}^{\frac{1}{2}}(\Gamma)}^{2} \leq c\left(\left\|I_{h}^{1} u_{1}^{h}\right\|_{H^{\frac{3}{2}}\left(\partial \Omega_{1}\right)}^{2}+\left\|\frac{\partial\left(I_{h}^{1} u_{1}^{h}\right)}{\partial \nu_{1}}\right\|_{H^{\frac{1}{2}}\left(\partial \Omega_{1}\right)}^{2}\right) \\
& \leq c\left\|I_{h}^{1} u_{1}^{h}\right\|_{H^{2}\left(\Omega_{1}\right)}^{2} \leq c\left|I_{h}^{1} u_{1}^{h}\right|_{H^{2}\left(\Omega_{1}\right)}^{2} \leq c\left|u_{1}^{h}\right|_{h, \Omega_{1}}^{2} \leq c A_{1}\left(u_{1}^{h}, u_{1}^{h}\right) .
\end{aligned}
$$

Construct the following continuous problem:

$$
\begin{cases}a\left(u_{2}, v\right)=0, & \forall v \in H_{0}^{2}\left(\Omega_{2}\right), \\ u_{2}=\frac{\partial}{\partial \nu_{2}} u_{2}=0, & \text { on } \partial \Omega_{2} \backslash \Gamma, \\ u_{2}=I_{h}^{1} u_{1}^{h}, \frac{\partial}{\partial \nu_{2}} u_{2}=-\frac{\partial}{\partial \nu_{1}}\left(I_{h}^{1} u_{1}^{h}\right), & \text { on } \Gamma .\end{cases}
$$

Note that $u_{2}^{h}$ is the Morley approximation of $u_{2}$. By Theorem 2.2, we obtain

$$
\begin{aligned}
A_{2}\left(u_{2}^{h}, u_{2}^{h}\right) & \leq 2\left(A_{2}\left(u_{2}, u_{2}\right)+A_{2}\left(u_{2}-u_{2}^{h}, u_{2}-u_{2}^{h}\right)\right) \\
& \leq c\left(\left\|u_{2}\right\|_{H^{2}\left(\Omega_{2}\right)}^{2}+h^{2}\left\|u_{2}\right\|_{H^{3}\left(\Omega_{2}\right)}^{2}\right) .
\end{aligned}
$$

The well-known a priori inequalities of the elliptic problem (2.10) yield [7, 9, 19]

$$
\begin{aligned}
\left\|u_{2}\right\|_{H^{2}\left(\Omega_{2}\right)}^{2} & \leq c\left(\left\|u_{2}\right\|_{H^{\frac{3}{2}}\left(\partial \Omega_{2}\right)}^{2}+\left\|\frac{\partial}{\partial \nu_{2}} u_{2}\right\|_{H^{\frac{1}{2}}\left(\partial \Omega_{2}\right)}^{2}\right), \\
\left\|u_{2}\right\|_{H^{3}\left(\Omega_{2}\right)}^{2} & \leq c\left(\left\|u_{2}\right\|_{H^{\frac{5}{2}}\left(\partial \Omega_{2}\right)}^{2}+\left\|\frac{\partial}{\partial \nu_{2}} u_{2}\right\|_{H^{\frac{3}{2}}\left(\partial \Omega_{2}\right)}^{2}\right) .
\end{aligned}
$$


Since $u_{2}$ and $\frac{\partial}{\partial \nu_{2}} u_{2}$ are piecewise polynomials on $\partial \Omega_{2}$, applying the fractional order inverse inequalities implied by the interpolation theorem of Sobolev spaces [1], we see that

$$
\left|u_{2}\right|_{H^{3}\left(\Omega_{2}\right)}^{2} \leq c h^{-2}\left(\left\|u_{2}\right\|_{H^{\frac{3}{2}}\left(\partial \Omega_{2}\right)}^{2}+\left\|\frac{\partial}{\partial \nu_{2}} u_{2}\right\|_{H^{\frac{1}{2}}\left(\partial \Omega_{2}\right)}^{2}\right) .
$$

With the above inequalities, we get

$$
\begin{aligned}
A_{2}\left(u_{2}^{h}, u_{2}^{h}\right) & \leq c\left(\left\|u_{2}\right\|_{H^{\frac{3}{2}}\left(\partial \Omega_{2}\right)}^{2}+\left\|\frac{\partial}{\partial \nu_{2}} u_{2}\right\|_{H^{\frac{1}{2}}\left(\partial \Omega_{2}\right)}^{2}\right) \\
& \leq c\left(\left\|I_{h}^{1} u_{1}^{h}\right\|_{H_{00}^{\frac{3}{2}}(\Gamma)}^{2}+\left\|\frac{\partial\left(I_{h}^{1} u_{1}^{h}\right)}{\partial \nu_{1}}\right\|_{H_{00}^{\frac{1}{2}}(\Gamma)}^{2}\right) .
\end{aligned}
$$

Consequently, (2.8) follows from (2.9) and (2.11), (2.7) can be established in the same manner.

Define the discrete extension operator $T_{h}^{k}: \Re^{I} \times \Re^{J} \rightarrow V_{h}^{k}$ as follows:

$\forall(\lambda, \mu) \in \Re^{I} \times \Re^{J}, T_{h}^{1}(\lambda, \mu) \in V_{h}^{1}: A_{1}\left(T_{h}^{1}(\lambda, \mu), v\right)=-A_{2}\left(E_{h}^{2}(\lambda, \mu), v\right), \forall v \in V_{h}^{0}$,

$\forall(\lambda, \mu) \in \Re^{I} \times \Re^{J}, T_{h}^{2}(\lambda, \mu) \in V_{h}^{2}: A_{2}\left(T_{h}^{2}(\lambda, \mu), v\right)=-A_{1}\left(E_{h}^{1}(\lambda, \mu), v\right), \forall v \in V_{h}^{0}$.

Corollary 2.5. Let $\Omega_{1}, \Omega_{2}, \sigma, \tau$ be the same as those in Theorem 2.4. For any $(\lambda, \mu) \in \Re^{I} \times \Re^{J}$, we have

$$
\frac{1}{\sigma} A_{2}\left(E_{h}^{2}(\lambda, \mu), E_{h}^{2}(\lambda, \mu)\right) \leq A_{1}\left(T_{h}^{1}(\lambda, \mu), T_{h}^{1}(\lambda, \mu)\right) \leq \tau A_{2}\left(E_{h}^{2}(\lambda, \mu), E_{h}^{2}(\lambda, \mu)\right),
$$

$$
\frac{1}{\tau} A_{1}\left(E_{h}^{1}(\lambda, \mu), E_{h}^{1}(\lambda, \mu)\right) \leq A_{2}\left(T_{h}^{2}(\lambda, \mu), T_{h}^{2}(\lambda, \mu)\right) \leq \sigma A_{1}\left(E_{h}^{1}(\lambda, \mu), E_{h}^{1}(\lambda, \mu)\right) .
$$

Proof. Let $(\lambda, \mu) \in \Re^{I} \times \Re^{J}$. Take $v \in V_{h}^{0}$ s.t. $v=E_{h}^{k}(\lambda, \mu)$ on $\Omega_{k}$. Then by the definition of $T_{h}^{1}$ and (2.7), we see that

$$
\begin{aligned}
A_{2}\left(E_{h}^{2}(\lambda, \mu), \quad E_{h}^{2}(\lambda, \mu)\right)=-A_{1}\left(T_{h}^{1}(\lambda, \mu), E_{h}^{1}(\lambda, \mu)\right) \\
\leq\left(A_{1}\left(T_{h}^{1}(\lambda, \mu), T_{h}^{1}(\lambda, \mu)\right)\right)^{\frac{1}{2}}\left(A_{1}\left(E_{h}^{1}(\lambda, \mu), E_{h}^{1}(\lambda, \mu)\right)\right)^{\frac{1}{2}} \\
\leq\left(A_{1}\left(T_{h}^{1}(\lambda, \mu), T_{h}^{1}(\lambda, \mu)\right)\right)^{\frac{1}{2}}\left(\sigma A_{2}\left(E_{h}^{2}(\lambda, \mu), E_{h}^{2}(\lambda, \mu)\right)\right)^{\frac{1}{2}} .
\end{aligned}
$$

So

$$
A_{1}\left(T_{h}^{1}(\lambda, \mu), T_{h}^{1}(\lambda, \mu)\right) \geq \frac{1}{\sigma} A_{2}\left(E_{h}^{2}(\lambda, \mu), E_{h}^{2}(\lambda, \mu)\right) .
$$

Take $v \in V_{h}^{0}$ s.t.

$$
v= \begin{cases}T_{h}^{1}(\lambda, \mu), & \text { on } \Omega_{1} \\ E_{h}^{2}\left(r_{0} T_{h}^{1}(\lambda, \mu), r_{1} T_{h}^{1}(\lambda, \mu)\right), & \text { on } \Omega_{2}\end{cases}
$$


Then, it follows from (2.8) and the definition of $T_{h}^{1}$ that

$$
\begin{aligned}
& A_{1}\left(T_{h}^{1}(\lambda, \mu), T_{h}^{1}(\lambda, \mu)\right)=-A_{2}\left(E_{h}^{2}(\lambda, \mu), E_{h}^{2}\left(r_{0} T_{h}^{1}(\lambda, \mu), r_{1} T_{h}^{1}(\lambda, \mu)\right)\right) \\
& \leq\left(A_{2}\left(E_{h}^{2}(\lambda, \mu), E_{h}^{2}(\lambda, \mu)\right)\right)^{\frac{1}{2}} \\
&\left(A_{2}\left(E_{h}^{2}\left(r_{0} T_{h}^{1}(\lambda, \mu), r_{1} T_{h}^{1}(\lambda, \mu)\right), E_{h}^{2}\left(r_{0} T_{h}^{1}(\lambda, \mu), r_{1} T_{h}^{1}(\lambda, \mu)\right)\right)\right)^{\frac{1}{2}} \\
& \leq\left(A_{2}\left(E_{h}^{2}(\lambda, \mu), E_{h}^{2}(\lambda, \mu)\right)\right)^{\frac{1}{2}} \\
&\left(\tau A_{1}\left(E_{h}^{1}\left(r_{0} T_{h}^{1}(\lambda, \mu), r_{1} T_{h}^{1}(\lambda, \mu)\right), E_{h}^{1}\left(r_{0} T_{h}^{1}(\lambda, \mu), r_{1} T_{h}^{1}(\lambda, \mu)\right)\right)\right)^{\frac{1}{2}} \\
&=\left(A_{2}\left(E_{h}^{2}(\lambda, \mu), E_{h}^{2}(\lambda, \mu)\right)\right)^{\frac{1}{2}}\left(\tau A_{1}\left(T_{h}^{1}(\lambda, \mu), T_{h}^{1}(\lambda, \mu)\right)\right)^{\frac{1}{2}} .
\end{aligned}
$$

Hence we have

$$
A_{1}\left(T_{h}^{1}(\lambda, \mu), T_{h}^{1}(\lambda, \mu)\right) \leq \tau A_{2}\left(E_{h}^{2}(\lambda, \mu), E_{h}^{2}(\lambda, \mu)\right) .
$$

Combining the above inequalities yields (2.12).

In the same manner, (2.13) can be established.

\section{Applications to domain decomposition analysis}

3.1. Domain decomposition method. The extension theorem plays a key role in the analysis of nonoverlapping, domain decomposition algorithms with two subdomains. As an example, define an algorithm as follows:

Step 1. Let $\left(\lambda^{0}, \mu^{0}\right) \in \Re^{I} \times \Re^{J}$ be given arbitrarily. Set $\mathrm{n}:=1$.

Step 2. Find $u_{1}^{n} \in V_{h}^{1}$ by solving the subproblem on $\Omega_{1}$ :

$$
\left\{\begin{array}{l}
A_{1}\left(u_{1}^{n}, v\right)=(f, v)_{1}, \forall v \in V_{h}^{1,0}, \\
r_{0} u_{1}^{n}=\lambda^{n-1} \\
r_{1} u_{1}^{n}=\mu^{n-1} .
\end{array}\right.
$$

Step 3. Find $u_{2}^{n} \in V_{h}^{2}$ by solving the subproblem on $\Omega_{2}$ :

$$
A_{2}\left(u_{2}^{n}, v\right)=-A_{1}\left(u_{1}^{n}, v\right)+(f, v), \quad \forall v \in V_{h}^{2} .
$$

Step 4. Select the relaxation factor $\theta_{n} \in(0,1)$ and calculate

$$
\lambda^{n}=\theta_{n} r_{0} u_{2}^{n}+\left(1-\theta_{n}\right) \lambda^{n-1}, \quad \mu^{n}=\theta_{n} r_{1} u_{2}^{n}+\left(1-\theta_{n}\right) \mu^{n-1} .
$$

Set $\mathrm{n}:=\mathrm{n}+1$, return to Step 2 until some reasonable stopping criterion is satisfied.

\subsection{Convergence analysis.}

Theorem 3.1. Let $u_{h}$ be the solution of (1.4). Let $u_{1}^{n}, u_{2}^{n}, \lambda^{n}, \mu^{n}$ be the values obtained by the algorithm in $\S 3.1$. Let $\varepsilon_{k}^{n} \in V_{h}^{k}$ s.t.

$$
\varepsilon_{k}^{n}(p)=u_{k}^{n}(p)-u_{h}(p), \frac{\partial \varepsilon_{k}^{n}}{\partial \nu}(m)=\frac{\partial u_{k}^{n}}{\partial \nu}(m)-\frac{\partial u_{h}}{\partial \nu}(m), \forall p, m \in \bar{\Omega}_{k} .
$$

Denote $\delta^{n}=\lambda^{n}-r_{0} u_{h}, \eta^{n}=\mu^{n}-r_{1} u_{h}$. Then,

$$
\frac{1}{\tau} A_{1}\left(\varepsilon_{1}^{n}, \varepsilon_{1}^{n}\right) \leq A_{2}\left(\varepsilon_{2}^{n}, \varepsilon_{2}^{n}\right) \leq \sigma A_{1}\left(\varepsilon_{1}^{n}, \varepsilon_{1}^{n}\right) .
$$

(2) There exists a constant $\theta^{*} \in(0,1]$, such that

$$
A_{1}\left(\varepsilon_{1}^{n+1}, \varepsilon_{1}^{n+1}\right) \leq \kappa\left(\theta_{n}\right) A_{1}\left(\varepsilon_{1}^{n}, \varepsilon_{1}^{n}\right),
$$

where $\kappa\left(\theta_{n}\right)<1, \forall \theta_{n} \in\left(0, \theta^{*}\right)$. 
There exists the optimal relaxation factor $\theta^{\text {opt }}$, such that

$$
\kappa\left(\theta^{o p t}\right)=\min _{\theta \in\left(0, \theta^{*}\right)} \kappa(\theta) .
$$

Proof. It is easy to see that $\varepsilon_{k}^{n+1} \in V_{h}^{k}$ satisfies

$$
\delta^{n+1}=\theta_{n+1} r_{0} \varepsilon_{2}^{n+1}+\left(1-\theta_{n+1}\right) \delta^{n}, \quad \eta^{n+1}=\theta_{n+1} r_{1} \varepsilon_{2}^{n+1}+\left(1-\theta_{n+1}\right) \eta^{n} .
$$

(3.4) and (3.5) yield

$$
A_{2}\left(\varepsilon_{2}^{n+1}, v\right)=-A_{1}\left(\varepsilon_{1}^{n+1}, v\right), \forall v \in V_{h}^{0} .
$$

Therefore $\varepsilon_{1}^{n+1}=E_{h}^{1}\left(\delta^{n}, \eta^{n}\right), \varepsilon_{2}^{n+1}=T_{h}^{2}\left(\delta^{n}, \eta^{n}\right)$. By Corollary 2.5, we get (3.1).

Furthermore, it follows from (3.4) and (3.6) that

$$
\begin{aligned}
\varepsilon_{1}^{n+1}=\theta_{n} & E_{h}^{1}\left(r_{0} \varepsilon_{2}^{n}, r_{1} \varepsilon_{2}^{n}\right)+\left(1-\theta_{n}\right) \varepsilon_{1}^{n}, \\
A_{1}\left(\varepsilon_{1}^{n+1}, \varepsilon_{1}^{n+1}\right)= & \theta_{n}^{2} A_{1}\left(E_{h}^{1}\left(r_{0} \varepsilon_{2}^{n}, r_{1} \varepsilon_{2}^{n}\right), E_{h}^{1}\left(r_{0} \varepsilon_{2}^{n}, r_{1} \varepsilon_{2}^{n}\right)\right) \\
& +2 \theta_{n}\left(1-\theta_{n}\right) A_{1}\left(E_{h}^{1}\left(r_{0} \varepsilon_{2}^{n}, r_{1} \varepsilon_{2}^{n}\right), \varepsilon_{1}^{n}\right) \\
& +\left(1-\theta_{n}\right)^{2} A_{1}\left(\varepsilon_{1}^{n}, \varepsilon_{1}^{n}\right) .
\end{aligned}
$$

By (2.7) and (3.1), we see that

$$
A_{1}\left(E_{h}^{1}\left(r_{0} \varepsilon_{2}^{n}, r_{1} \varepsilon_{2}^{n}\right), E_{h}^{1}\left(r_{0} \varepsilon_{2}^{n}, r_{1} \varepsilon_{2}^{n}\right)\right) \leq \sigma A_{2}\left(\varepsilon_{2}^{n}, \varepsilon_{2}^{n}\right) \leq \sigma^{2} A_{1}\left(\varepsilon_{1}^{n}, \varepsilon_{1}^{n}\right) .
$$

(3.7) gives $A_{2}\left(\varepsilon_{2}^{n+1}, \varepsilon_{2}^{n+1}\right)=-A_{1}\left(\varepsilon_{1}^{n+1}, E_{h}^{1}\left(r_{0} \varepsilon_{2}^{n+1}, r_{1} \varepsilon_{2}^{n+1}\right)\right)$. So by (3.1), we get

$$
A_{1}\left(E_{h}^{1}\left(r_{0} \varepsilon_{2}^{n}, r_{1} \varepsilon_{2}^{n}\right), \varepsilon_{1}^{n}\right)=-A_{2}\left(\varepsilon_{2}^{n}, \varepsilon_{2}^{n}\right) \leq-\frac{1}{\tau} A_{1}\left(\varepsilon_{1}^{n}, \varepsilon_{1}^{n}\right) .
$$

If $0<\theta_{n}<1$, then (3.2) follows from (3.8), (3.9) and (3.10). Furthermore, $\kappa\left(\theta_{n}\right)$ has the following expression:

$$
\kappa\left(\theta_{n}\right)=\frac{1}{\tau}\left(\theta_{n}^{2}\left(\sigma^{2} \tau+\tau+2\right)-2 \theta_{n}(\tau+1)+\tau\right) .
$$

An elementary calculation indicates $0 \leq \kappa\left(\theta_{n}\right)<1$, if and only if

$$
0<\theta_{n}<\theta^{*}=\min \left(1, \frac{2(\tau+1)}{\sigma^{2} \tau+\tau+2}\right) .
$$

The optimal relaxation factor is given by

$$
\theta^{o p t}=\frac{\tau+1}{\sigma^{2} \tau+\tau+2},
$$

for which

$$
\kappa\left(\theta^{o p t}\right)=\frac{\sigma^{2} \tau^{2}-1}{\tau\left(\sigma^{2} \tau+\tau+2\right)}=\min _{\theta \in\left(0, \theta^{*}\right)} \kappa(\theta) .
$$

So (3.3) follows from (3.13). 
The algorithm in $\S 3.1$ is the generalization of the so-called Dirichlet-Neumann alternative method or the Marini-Quarteroni method [17] to plate bending problems. Its essence lies in the continuing correction of the initial guess of $\left(r_{0} u_{h}, r_{1} u_{h}\right)$ by imposing $r_{0}\left(\triangle u_{1}^{n}\right)=r_{0}\left(\triangle u_{2}^{n}\right)$ and $r_{1}\left(\Delta u_{1}^{n}\right)=r_{1}\left(\triangle u_{2}^{n}\right)$ at each iteration. Theorem 3.1 implies that the algorithm in $\S 3.1$ converges geometrically and independently of $h$, which is guaranteed by the extension theorem (Theorem 2.4). In the special case that the domain $\Omega$ is symmetric with respect to $\Gamma$, then $\sigma=\tau=1$ in (2.7) and (2.8); thus, by (3.12) and (3.13), $\theta^{\text {opt }}=\frac{1}{2}$ and $\kappa\left(\theta^{\text {opt }}\right)=0$, which together with (3.3) show that only one iteration is needed to obtain the solution of (1.4).

Of course, other algorithms in [11] can be generalized and their analysis can be carried out similarly, based on Theorem 2.4.

3.3. Numerical experiments. Decompose the domain $\Omega=(0,1.5) \times(0,1) \cup(0,1)$ $\times[1,2)$ into subdomains: $\Omega_{1}=(0,1) \times(1,2), \Omega_{2}=(0,1.5) \times(0,1)$. Triangulate $\Omega$ to get the fine mesh $\Omega_{h}$ so that each element $e \in \Omega_{h}$ is an isosceles right triangle with $h$ as its diameter. When $h=0.25$, there are 32, 48 elements and 81, 117 interpolation points in $\Omega_{1}, \Omega_{2}$, respectively. When $h=0.125$, there are 128, 192 elements and 289,425 interpolation points in $\Omega_{1}, \Omega_{2}$, respectively. When $h=0.0625$, there are 512,768 elements and 1089, 1617 interpolation points in $\Omega_{1}, \Omega_{2}$, respectively. In the above three cases, there are 7, 15 and 31 interpolation points on $\Gamma$ respectively. For an edge midpoint $m$, if $m \in \partial e_{1} \cap \partial e_{2}$, then the outward normal vectors of $e_{1}$ and $e_{2}$ at $m$ are opposite. To ensure that $\frac{\partial v}{\partial \nu}(m)$ are determined uniquely, we require that the outward (inward) normal vectors be chosen for the triangular elements with even (odd) numbers. In the following tables, $n$ is the number of iterations, $\varepsilon^{n}$ is the error after $n$ iterations, $\left\|\varepsilon^{n}\right\|_{A}=A\left(\varepsilon^{n}, \varepsilon^{n}\right), \rho_{n}=\sqrt[n]{\left\|\varepsilon^{n}\right\|_{A} /\left\|\varepsilon^{0}\right\|_{A}}$ and $\left\|\varepsilon^{n}\right\|_{\infty}=\left\|\varepsilon^{n}\right\|_{L^{\infty}(\Omega)}$.

When using the algorithm of $\S 3.1$ to solve (1.4), a procedure is built up to generate a sequence of the discrete biharmonic functions on $\Omega_{1}$ and $\Omega_{2}$ with the same values at $p, m \in \Gamma$. This allows us to compute, at each iteration, two constants $\sigma_{n}, \tau_{n}$ suggested by (2.7) and (2.8), which combined with (3.12) gives the sequence of approximate values $\theta_{n}$ of the optimal relaxation factor $\theta^{\text {opt }}$. We point out that the evaluation of $\theta_{n}$ does not require the solution of any additional problem in our algorithm (for details, cf. [17] ). The main experimental results, obtained on a SGI work station, are listed in Table 1 and Table 2, and support our theoretical analysis.

TABLE 1. Error reduction factor $\rho_{n}$ vs. $h$

\begin{tabular}{|c|c|c|c|c|}
\hline$n$ & 1 & 5 & 9 & 13 \\
\hline$h=0.2500$ & 0.0253 & 0.0343 & 0.0261 & 0.0395 \\
\hline$h=0.1250$ & 0.0272 & 0.0476 & 0.0438 & 0.0558 \\
\hline$h=0.0625$ & 0.0264 & 0.0513 & 0.0473 & 0.0464 \\
\hline
\end{tabular}

TABLE 2. The errors $\left\|\varepsilon^{n}\right\|_{A}$ and $\left\|\varepsilon^{n}\right\|_{\infty}$ when $h=0.0625$

\begin{tabular}{|c|c|c|c|c|}
\hline$n$ & 1 & 5 & 9 & 13 \\
\hline$\left\|\varepsilon^{n}\right\|_{A}$ & $0.487 \cdot 10^{9}$ & $0.129 \cdot 10^{4}$ & $0.532 \cdot 10^{-1}$ & $0.718 \cdot 10^{-6}$ \\
\hline$\left\|\varepsilon^{n}\right\|_{\infty}$ & $0.952 \cdot 10^{4}$ & $0.837 \cdot 10^{2}$ & $0.261 \cdot 10^{0}$ & $0.459 \cdot 10^{-3}$ \\
\hline
\end{tabular}




\section{EXTENSION THEOREMS FOR OTHER PLATE ELEMENTS}

Let $V_{h}$ be Fraeijs de Veubeke element space $\mathcal{F}_{h}$ or Zienkiewicz element space $\mathcal{Z}_{h}$ or Adini element space $\mathcal{A} D_{h}$ described in [4]. As in Sect. 2, we can define the discrete biharmonic operator $E_{h}^{k}: \Re^{M} \rightarrow V_{h}^{k}$ correspondingly. Here $M$ denotes the number of degrees of freedom associated with the interface $\Gamma$.

Theorem 4.1. (Extension theorem) Let $\Omega_{1}, \Omega_{2}$ be convex. If $V_{h}$ is one of the three nonconforming plate element spaces $\mathcal{F}_{h}, \mathcal{Z}_{h}$ and $\mathcal{A} D_{h}$, there exist two constants $\hat{\sigma}, \hat{\tau}$, independent of the quasi-uniform mesh parameter $h$, such that

$$
\hat{\sigma}=\sup _{\eta \in \Re^{M}} \frac{A_{1}\left(E_{h}^{1} \eta, E_{h}^{1} \eta\right)}{A_{2}\left(E_{h}^{2} \eta, E_{h}^{2} \eta\right)}<\infty, \quad \hat{\tau}=\sup _{\eta \in \Re^{M}} \frac{A_{2}\left(E_{h}^{2} \eta, E_{h}^{2} \eta\right)}{A_{1}\left(E_{h}^{1} \eta, E_{h}^{1} \eta\right)}<\infty .
$$

We can adopt the ideas of the proof of Theorem 2.4 to prove Theorem 4.1 with the following points in mind:

1. The error of the nonconforming approximate solution of the inhomogeneous boundary value problem can be estimated by first obtaining an inequality similar to (2.1), subtracting off appropriate "conforming" parts as in (2.2) and then applying the bilinear lemma [6], cf. [3], [6], [15], [22].

2. The conforming interpolation operator $I_{h}^{k}$ must be constructed by similarly modifying the corresponding one introduced in [4] and Theorem 2.3 still holds in this case.

Theorem 4.2. (Extension theorem) Let $\Omega_{1}, \Omega_{2}$ be convex. If $V_{h}$ is one of the conforming plate element spaces [6], then (4.1) holds.

Since it is unnecessary to construct the conforming interpolation operator $I_{h}^{k}$ in this case, the proof of Theorem 4.2 is much simpler than the proof of Theorem 2.4, so we omit it here. Analogous results may be found in [24].

Further applications of these extension theorems will be given in forthcoming papers.

\section{ACKNOWLEDGEMENTS}

The authors are indebted to Professor Wang Lie-heng, Professor Yu De-hao, Dr. Chu De-lin and Dr. Xie Zheng-hui for their helpful comments on an early version of this paper, particularly Dr. Huang Jian-guo for many fruitful discussions on Sobolev spaces on the boundary of the polygonal domain and finally the referees for their beneficial advice and suggestions.

\section{REFERENCES}

[1] R.A. Adams, Sobolev Spaces, Academic Press, New York, 1975. MR 56:9247

[2] J.H. Argyris, I. Fried and D.W. Scharpf, The TUBA family of plate elements for the matrix displacement method, Aero. J. Roy. Aero. Soc. 72(1968), 701-709.

[3] D.N. Arnold and F. Brezzi, Mixed and nonconforming finite element methods: implementation, postprocessing and error estimates, M²AM. 19(1985), 7-32. MR 87g:65126

[4] S.C. Brenner, A two-level additive Schwarz preconditioner for nonconforming plate elements, Numer. Math. 72(1996), 419-447. CMP 96:08

[5] T.F. Chan, W. E and J. Sun, Domain decomposition interface preconditioners for fourth order elliptic problems, Appl. Numer. Math. 8(1991), 317-331. MR 92g:65125

[6] P.G. Ciarlet, The Finite Element Method for Elliptic Problems, North-Holland, Amsterdam, 1978. MR 58:25001 
[7] M. Dauge, Elliptic Boundary Value Problems on Corner Domains: Smoothness and Asymptotics of Solutions, Springer-Verlag, New York, 1988. Lecture Notes in Math., Vol.1341. MR 91a:35078

[8] M. Dryja and O.B. Widlund, An additive variant of the Schwarz alternating method in the case of many subregions, Technical Report 339, Dept. of Computer Science, Courant Institute, 1987.

[9] P. Grisvard, Elliptic Problems in Nonsmooth Domains, Pitman, Boston, 1985. MR 86m:35044

[10] J. Gu, Domain Decomposition Methods with Nonconforming Finite Elements, Ph.D. thesis (in Chinese), Dept. of Applied Math., Tsinghua University, China, 1994.

[11] J. Gu and X. Hu, On domain decomposition methods in two-subdomain nonoverlap cases, Chinese J. Num. Math. \& Appl. 17:1(1995), 78-94.

[12] _ On an essential estimate in the analysis of domain decomposition methods, J. Comput. Math. 12:2(1994), 132-137. MR 95a:65205

[13] _ Some estimates with nonconforming finite elements in domain decomposition analysis, J. Comput. Math. 15:3(1997).

[14] - Trace averaging domain decomposition method with nonconforming finite elements, J. Comput. Math. 14:1(1996), 40-53. MR 96k:65078

[15] P. Lascaux and P. Lesaint, Some nonconforming finite elements for the plate bending problem, RAIRO. R-1(1975), 9-53. MR 54:11941

[16] J.L. Lions and E. Magenes, Non-homogeneous Boundary Value Problems and Applications.I, Grund. B. 181. Berlin, Springer, 1972. MR 50:2670

[17] L.D. Marini and A. Quarteroni, A relaxation procedure for domain decomposition methods using finite elements, Numer. Math. 55(1989), 575-598. MR 90g:65150

[18] L.S.D. Morley, The triangular equilibrium problem in the solution of plate bending problems, Aero. Quart. 19(1968), 149-169.

[19] J. Nečas, Les Méthodes Directes en Théorie des Équations Elliptiques, Academia, Prague, 1967. MR 37:3168

[20] P. Oswald, Hierarchical conforming finite element methods for the biharmonic equation, SIAM J. Numer. Anal. 29(1992), 1610-1625. MR 93k:65098

[21] Z. Shi, Error estimates for the Morley element, Chinese J. Num. Math. \& Appl. 12:3(1990), 102-108. MR 91i:65182

[22] F. Stummel, Basic compactness properties of nonconforming and hybrid finite element spaces, RAIRO Anal. Num. 4:1(1980), 81-115. MR 81h:65058

[23] J. Sun, Multilevel preconditioners for 4th order problems and domain decomposition methods, In Proceedings of the Sixth International Conference on Domain Decomposition, A. Quarteroni et al (eds.) Italy, 1992.

[24] O.B. Widlund, An extension theorem for finite element spaces with three applications, in Numerical Techniques in Continuum Mechanics, Vol.16. Hackbusch. W.(eds). Braaunschweig Wiesbaden, 1987.

[25] X. Zhang, Studies in Domain Decomposition: Multilevel Methods and the Biharmonic Dirichlet Problem, Ph.D. thesis (Technical Report 584), Courant Institute, New York University, 1991.

[26] _ Multilevel Schwarz methods for the biharmonic Dirichlet problem, SIAM J. Sci. Comput. 15:3(1994), 621-644. MR 96a:65048

[27] Two-level Schwarz methods for the biharmonic problem discretized by conforming $C^{1}$ elements, SIAM J. Numer. Anal. 33(1996), 555-570. MR 97b:65133

Department of Mathematics, Capital Normal University, Beijing 100037, China

E-mail address: gjs@mailhost.cnu.edu.cn

Department of Applied Mathematics, Tsinghua University, Beijing 100084, China 